\title{
L'éducation pour une citoyenneté démocratique au niveau du Curriculum scolaire en Roumanie
}

Ion Albulescu

\section{OpenEdition}

1 Journals

Édition électronique

URL : http://journals.openedition.org/trema/350

DOI : 10.4000/trema.350

ISSN : 2107-0997

Éditeur

Faculté d'Éducation de l'université de Montpellier

\section{Édition imprimée}

Date de publication : 1 mars 2007

Pagination : 5-14

ISSN : 1167-315X

\section{Référence électronique}

Ion Albulescu, «L'éducation pour une citoyenneté démocratique au niveau du Curriculum scolaire en Roumanie », Tréma [En ligne], 27 | 2007, mis en ligne le 20 janvier 2010, consulté le 19 avril 2019. URL http://journals.openedition.org/trema/350 ; DOI : 10.4000/trema.350

Ce document a été généré automatiquement le 19 avril 2019

Trema 
L'éducation pour une citoyenneté démocratique au niveau du Curriculum scolaire en Roumanie

Ion Albulescu

I. Introduction : l'éducation pour une citoyenneté démocratique 
1 Le système d'enseignement est perçu comme un service social qui doit accomplir des fonctions complexes. Il va chercher à former les élèves en fonction de diverses activités, pour développer le côté spirituel de la vie, pour les former en tant que citoyens. En se chargeant de la formation des citoyens, l'école doit s'adapter à travers des projets curriculaires novateurs : prenant en compte les changements

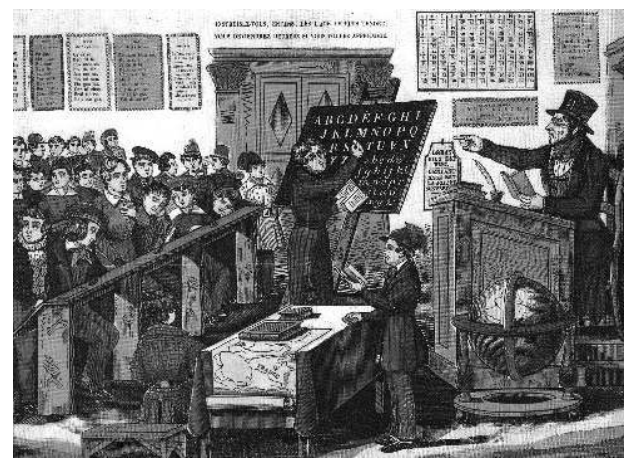
rapides dont on parle au niveau de la société. Les mutations intervenues dans le monde contemporain ont généré une nouvelle problématique pour l'éducation, centrée autour d'une série de valeurs considérées comme prioritaires : démocratie, participation, responsabilité, tolérance, coopération, communication etc. Les développements théoriques de cette question et les démarches pratiques demandés sont contenus dans le syntagme "Education pour une citoyenneté démocratique ». Bien que celle - ci ait été assidûment promue depuis longtemps par l'intermédiaire des programmes éducatifs, l'éducation pour une citoyenneté démocratique a été imposée en Roumanie par le Curriculum scolaire, constitué après 1990. A cela s'ajoute, toute une série d'actions concernant l'éducation des adultes, par les activités éducatives non formelles et formelles.

2 L'éducation pour une citoyenneté démocratique dans le système roumain d'enseignement se réalise conformément à la Convention des chefs d'Etats et de gouvernement (Vienne, 8 - 9 oct. 1993) et aux directives adoptées à la Conférence des ministres d'éducation européens (Madrid, 23 - 24 mars, 1994). Ces documents proposent une acception consensuelle pour le concept d'éducation, comprenant :

- a) Un positionnement contre celui qui nie les valeurs de la démocratie (par exemple : l'extrémisme caractérisé par xénophobie, racisme, violence etc.).

- b) Une préoccupation pour la vie personnelle des individus en relation avec les contextes sociopolitiques.

- c) Une voie pour le développement de la société démocratique par l'encouragement de la réflexion critique, l'admission des points de vue différents, l'apprentissage de stratégies pour élaborer des solutions non - violentes aux conflits.

3 Cette éducation a pour but la formation et le développement chez l'individu des compétences afin de pouvoir participer d'une manière active à la vie publique (participation aux élections, interventions pour influencer les décisions politiques, défense mise en pratique de ses droits, comportement adéquat et responsable dans la société). La pratique de la citoyenneté démocratique représente un engagement citoyen face à l'autorité publique, sans aucune discrimination. Nous avons, dans ce cas - là, un pouvoir légitime de décider et d'agir d'une manière volontaire. La démocratie participative est, selon J. DEWEY (1990), plus qu'une simple forme de gouvernement. Celle - ci est, en premier lieu, une façon de vivre, par l'intermédiaire des expériences communiquées et partagées. Autrement dit, la démocratie est un mode de vie. Les gens délibèrent ensemble sur les problèmes et sur les projets qu'ils partagent. Les décisions sont prises par ceux qui sont directement affectés de leurs conséquences. Cette vision a, de toutes façons, ses propres limites. Une grande partie de la population dispose d'une série de droits, mais il est impossible que tous les gens participent activement et 
directement à la prise de toutes les décisions qui les concernent. En conséquence, nous assistons à un remplacement de l'autorité par des personnes représentatives, acte qui suppose de la part des citoyens des connaissances, liberté de s'exprimer et responsabilité. En perspective éducative, la formation des individus pour une société démocratique signifie non seulement l'acquisition des connaissances sur les mécanismes de la démocratie, mais aussi la formation à de nouvelles compétences et attitudes. L'éducation pour la citoyenneté démocratique promeut la culture de la démocratie, la répartition des responsabilités, l'intercompréhension, l'apaisement des conflits. Ses objectifs majeurs sont la transmission d'un système de valeurs (pluralisme, les droits de l'homme, coopération, participation, tolérance, respect etc.), la formation d'une capacité à dialoguer, à changer et à prendre des décisions responsables, la formation des attitudes positives et aussi la capacité à assumer des responsabilités au sein de la communauté, de faire preuve de solidarité et de confiance, de témoigner d'une certaine flexibilité en relation avec les autres, de respect envers leur culture, de trouver une solution non violente aux situations qui posent problème.

4 L'éducation pour une citoyenneté démocratique nous apparait comme un ensemble de pratiques et d'activités dont le principal but est la formation des jeunes et des adultes en vue de participer activement à la vie de la cité, par l'acceptation et la mise en pratique des droits et des obligations (responsabilités) envers leur société. L'éducation pour cette citoyenneté n'est ni une discipline scolaire, ni une autre activité prévue dans le curriculum, ni un domaine de connaissances, ni une forme d'action sociale, ni un type d'éducation synonyme de l'éducation des droits de l'homme, de l'éducation politique ou de l'éducation des élites, mais un objectif majeur des politiques éducatives, conçu dans la perspective de l'apprentissage continuel et qui subordonne toutes les dimensions antérieurement énoncées.

5 Nous pouvons affirmer qu'il existe trois moyens de réalisation de l'éducation pour une citoyenneté démocratique à l'école :

- a) les moments, les endroits et les occasions où les élèves peuvent prendre des initiatives, en dehors des activités d'enseignement proprement dites ;

- b) la vie scolaire quotidienne et, plus précisément, toutes les situations créées par l'école en tant que milieu social, avec ses règles collectives, les conflits interpersonnels, les moments et les occasions de coopération ;

- c) les leçons proprement dites, par lesquelles se réalisent l'enseignement et l'apprentissage des disciplines qui ont des objectifs caractéristiques à la société démocratique.

6 Par la multitude d'interrelations qu'elle crée et stimule, ce type d'éducation favorise un apprentissage qui s'étend au - delà du cadre formel des activités curriculaires. Toute la structure organisationnelle de l'école est déterminée par son rôle essentiel, la réalisation de l'éducation pour une citoyenneté démocratique.

\section{L'apprentissage des comportements démocratiques dans l'école}

7 L'apprentissage des élèves de comportements démocratiques ne peut se réaliser que dans un milieu éducatif adéquat. Généralement, on considère que les enseignants qui ont une attitude autoritaire à l'égard de leurs élèves assument entièrement les décisions ayant 
trait aux processus de formation et d'éducation : des formes d'organisation, d'emploi du temps, modalités à aborder les tâches etc. Ils apprécient la direction excessive et l'ordre rigide, ainsi que l'obéissance et la sanction.

Ce sont eux, ceux qui imposent la conduite. La curiosité et la spontanéité des élèves sont assez souvent confondues avec l'impertinence et le non - respect des règles, qui doivent être sanctionnés. Etant convaincus qu'il est nécessaire d'adopter une attitude sévère et intolérante à l'égard de la conduite des élèves, les enseignants tentent d'exercer un contrôle continu et rigoureux, en n'admettant aucune déviation du règlement. Leurs connaissances, opinions et décisions sont incontestables.

Les pédagogues réclament tous le fait que l'encouragement d'une telle méthode de travail, présente dans l'école traditionnelle provoque, en ce qui concerne les élèves, le conformisme, l'adaptation passive, l'attitude non critique, la peur de l'échec qui, plus tard, affecteront la participation réelle à la vie de la société démocratique. Il est sûr qu'il s'agit d'une démarche négative de la part des enseignants qui ne doit pas être tolérée dans une école moderne.

Nous pouvons penser à une autre signification de l'autorité des professeurs. Il faut la percevoir en tant que mise en pratique de leur capacité à accomplir avec succès le rôle assumé, situation qui évite le changement de l'autorité en autoritarisme. Nous ne devons pas confondre l'autorité avec l'attitude dictatoriale, car celle - ci exclut la répression arbitraire, en s'appuyant sur le consentement, et non pas sur la contrainte. Il ne faut pas l'associer a priori à la sévérité rigide. Il convient de la mettre en relation avec la fermeté et l'efficacité.

11 Le professeur ne peut faire preuve de son autorité que devant les élèves libres et responsables. L'accentuation de la responsabilité au détriment de la liberté mène à la dictature. De même, l'accentuation de la liberté au détriment de la responsabilité conduit au chaos. Les deux situations représentent, finalement, une négation de l'autorité. L'enseignant fait remarquer son autorité en vertu de son statut et des compétences prouvées, en obtenant la reconnaissance de la part des élèves non par l'intermédiaire de la contrainte, mais à travers le consentement volontairement exprimé. Plus les idées, les normes ou les directives contribuent à obtenir le consentement des élèves, plus l'exercice de l'autorité est authentique. De cette façon, l'autorité ne devient pas une menace, mais une forme de directive pertinente et efficace des efforts d'apprentissage des élèves, directive qui détermine l'autonomie de sa pensée, l'initiative, l'implication, la coopération, la décision autonome, celles - ci se constituant toutes en qualités indispensables à l'individu, afin d'exercer la citoyenneté démocratique. En agissant d'une manière ferme et flexible à la fois, même ouverte à la pluralité des idées et des actions, les professeurs peuvent contribuer à la formation des personnalités dynamiques, créatives, capables de répondre aux exigences complexe de la société démocratique.

\section{Les projections curriculaires}

Après 1990, le système d'enseignement roumain a été soumis à une réforme visant à le libéraliser. Il est significatif que l'éducation pour une citoyenneté démocratique a été introduite en tant que composante essentielle du Curriculum scolaire. Dans ce cadre l'éducation pour une citoyenneté démocratique est introduite par l'intermédiaire des disciplines Education civique (les classes IIIe - IVe de l'école primaire) et Culture civique (le 
collège), à l'aide desquelles on acquiert des connaissances sur la société, les droits de l'homme, la démocratie, le système politique etc. Le motif pour lequel on a introduit dans l'école de telles disciplines réside dans le fait que le petit écolier est impliqué lui aussi dans cette éducation, grâce à son appartenance à la famille, aux groupes de jeu et d'enseignement et grâce aux liens spécifiques qu'il peut établir avec diverses institutions et $\mathrm{L}$ éducation pour une citoyenneté démocratique organisations de la communauté où il vit (l'école, l'église, la mairie, les organisations non gouvernementales etc.). Par la suite, le processus de socialisation peut et doit commencer tôt. L'instruction réalisée par ces disciplines répond à un besoin pratique d'intégration et d'action dans la société (un comportement adéquat dans la société démocratique), en concordance avec ses valeurs. L'introduction des cours d'Education civique dans les classes primaires représente un commencement de responsabilité sociale de l'écolier, un changement qui peut contribuer à la réalisation d'une conduite sociale désirable, du civisme et à l'implication dans la communauté.

Le Curriculum pour la discipline Education civique (IIIe et IVe classes primaires) a été élaboré en tenant compte des exigences formulées dans le texte de la Loi de l'Enseignement, qui concerne l'idéal éducatif et les finalités de l'enseignement. Dans l'enseignement primaire, les classes IIIe et IVe, la place de la discipline Education civique dans le Curriculum scolaire est justifiée par :

- a) le besoin d'initier les petits écoliers à la pratique d'un comportement civique dans une société démocratique, un comportement actif, responsable, tolérant, ouvert, communicatif, attentif, auto - évaluatif ;

- b) la nécessité d'une alphabétisation civique des écoliers, par la familiarisation de ceux - ci avec le langage, les sujets et les activités d'apprentissage spécifiques ;

- c) la possibilité de valoriser l'expérience spécifique à l'âge par l'accentuation des dimensions affectives et de comportement associé à celles qui concernent la stimulation de la participation et de la communication responsables.

Les contenus de l'enseignement et de l'apprentissage de la discipline Education civique (IIIe), par exemple, sont constitués des connaissances se rapportant à la personne, ses qualités morales (la confiance / le manque de confiance de soi et de l'autre, le respect / le manque de respect, le courage, la lassitude, la bonté, la méchanceté, la sincérité, le mensonge), les rapports de l'individu avec les choses et les êtres (besoins, attitudes etc.), les rapports avec ses proches (les groupes d'appartenance : la famille, le groupe à jouer, le groupe à apprendre, le groupe religieux), les règles du groupe auquel il appartient, ses droits et ses obligations Pour la IVe, les contenus d'enseignement sont centrés sur les sujets suivants : nous et les autres, la communauté, la société et l'Etat. Pour établir ces contenus, on a eu comme point de départ l'idée conformément à laquelle le sujet central est l'individu, analysé à travers des qualités, des valeurs et des attitudes désirables. On insiste sur la problématique de la personne seulement dans les classes primaires, car, dans les classes de collège, l'accent se déplace sur l'Etat, sur la société civile sur les droits et sur les obligations des citoyens. En même temps, les contenus de cette discipline vont diriger l'élève vers la connaissance et la compréhension des problèmes de la communauté, comme un pas important vers la voie de sa socialisation.

15 A partir de l'année scolaire 2003 - 2004, les programmes scolaires de Culture civique pour les classes Ve et VIe du collège peuvent être utilisés dans le système d'enseignement comme offre pour le Curriculum à la Décision de l'Ecole. Ces programmes représentent une offre curriculaire d'enseignement apprentissage axée sur l'éducation morale et civique. 
En même temps, dans le programme pour la Ire classe du collège (Ve en Roumanie), on insiste sur le fait que la démarche de familiarisation avec le spécifique de l'éducation civique, esquissé dans les classes primaires, est reprise et approfondie par :

- a) des connaissances sur les valeurs et les normes morales, les qualités morales de la personne, les attitudes et les comportements moraux;

- b) la compréhension du spécifique des normes morales;

- c) la réflexion critique sur les valeurs, les normes et les comportements moraux ;

- d) la formation des attitudes et des comportements moraux, leur mise en pratique dans le cadre des activités d'apprentissage.

Dans la perspective de l'intégration de la Roumanie dans la Communauté Européenne, par l'enseignement et l'apprentissage de la discipline Culture civique, on essaie non seulement d'expliciter des concepts spécifiques, mais aussi de développer certaine manière de penser, d'une capacité augmentée de percevoir la réalité de la vie nationale et internationale, de la volonté d'agir pour édifier un monde où les droits de l'homme, de l'enfant soient reconnus et respectés. D'ailleurs, la discipline contribue à l'initiation et au développement d'un partenariat entre l'école et la société civile qui offre le support pour le développement de la citoyenneté active dans le contexte des réponses adéquates aux besoins de la communauté.

Le programme curriculaire pour la seconde classe du collège (VIe en Roumanie) est conçu de telle sorte que la démarche de familiarisation des élèves avec le spécifique de l'éducation civique, esquissée dans les IIIe, IVe et Ve classes, soit reprise et approfondie par:

- a) des connaissances sur les droits fondamentaux de l'enfant, sur les institutions et les organisations qui s'occupent de la promotion et de l'observation de ceux - ci ;

- b) la reconnaissance des principes et des droits de l'enfant ;

- c) la pensée critique à l'égard du rapport qui s'établit entre les droits et les responsabilités et à l'égard des situations de respect / violation de ceux - ci ;

- d) la formation des attitudes et des comportements démocratiques, la mise en pratique dans le cadre des activités d'apprentissage qui ont comme point de référence les modèles démocratiques.

Les contenus de la discipline Culture civique (VIe classe) sont constitués des connaissances sur l'enfant et sur l'univers de l'enfance, sur les droits de l'homme et de l'enfant, sur des documents internationaux ayant trait à ces droits et aux principes sur lesquels ils sont fondés, sur les modalités de promotion et de défense des droits de l'enfant etc.

La discipline Culture civique est aussi prévue dans le Programme d'enseignement pour les VIIe et VIIIe classes du collège (une heure par semaine), sa place se justifie par le besoin de continuer et d'approfondir l'éducation civique des élèves, initiée dans les années L éducation pour une citoyenneté démocratique antérieures, en ce qui concerne la pratique d'un comportement civique, défini par des traits comme: la responsabilité, la tolérance, la communication etc. La démarche de familiarisation avec la spécificité de l'éducation civique, esquissée déjà dans les classes primaires, est reprise d'une manière concentrique et approfondie par :

- a) des connaissances sur les personnes, sur la société et sur l'Etat ;

- b) la transmission des valeurs et des normes sociales;

- c) la pensée critique sur les valeurs et les normes sociales ; 
- d) la formation des attitudes et des comportements, la mise en pratique de ceux - ci dans le cadre des activités d'apprentissage qui ont comme référence les pratiques démocratiques.

20 La Culture civique pour les VIIe et VIIIe classes est conçue comme une continuation des éléments acquis dans les années antérieures. Cette discipline est entendue comme « une démarche interdisciplinaire et interculturelle, qui réalise ouverture, communication et flexibilité, qui tient compte des expériences des élèves et qui permet la valorisation positive de celle - ci » (L. Vl_sceanu, coord., 2002, p. 215). Les contenus de l'enseignement et de l'apprentissage de la discipline Culture civique dans la VIIe classe sont constitués des connaissances se rapportant à la vie sociale, aux attitudes et aux relations interpersonnelles dans le cadre du groupe, à la famille, aux droits de l'homme, au système politique de notre pays, aux institutions et aux pratiques démocratiques, aux autorités de l'Etat, à la relation citoyen - Etat, aux droits et aux devoirs des citoyens etc. Dans la VIIIe classe, par l'intermédiaire des principes et concepts essentiels de la démocratie (par exemple, l'autorité, la responsabilité, la justice, la liberté, la propriété etc.) sont suivies des discussions nuancées sur l'analyse des valeurs / principes de la démocratie et la mise en pratique des principes du régime politique démocratique, avec lequel les élèves sont déjà habitués. Les contenus de l'enseignement et de l'apprentissage de la discipline Culture civique (VIIIe classe) sont constitués des connaissances qui se rapportent à l'autorité dans l'espace public et privé, aux relations entre les citoyens et l'autorité, aux conséquences du manque ou de l'abus de l'autorité, à la liberté et à la responsabilité, à la justice et à l'égalité, à la propriété, au patriotisme et à l'intégration européenne etc.

21 Les sujets prévus dans les programmes de Culture civique pour les VIIe et VIIIe classes concordent avec les idéaux de notre société: égalité, différence et compétition, pluralisme, tolérance, propriété privée et économie de marché, la complexité de l'identité personnelle, l'intégration et l'acceptation dans un espace spirituel plus large qui dépasse les frontières nationales etc.

22 A part les disciplines dont on a déjà parlé, qui sont étudiées dans l'enseignement primaire et au collège, le présent curriculum contient pour l'enseignement au lycée des disciplines qui peuvent contribuer, à leur tour, à l'éducation pour une citoyenneté démocratique : sociologie, économie, philosophie, histoire, géographie etc. L'orientation d'ensemble n'était pas seulement d'introduire les objectifs spécifiques à l'éducation pour une citoyenneté démocratique dans le programme d'autres disciplines, mais aussi d'offrir des connaissances sur l'homme et sur la nature. Dans la perspective des comportements et des relations qui s'établissent entre les individus, un grand nombre de disciplines attirent l'attention sur le développement des compétences qui rendent possible le dialogue, la participation et la responsabilité. Par la suite, les sciences humaines jouent un rôle essentiel. En étudiant les sociétés passées et présentes, celles - ci créent des modalités de compréhension de la vie sociale, économique, politique et culturelle, des modalités qui incluent une dimension historique et une dimension territoriale qui sont mises en relation avec les valeurs et les conceptions humaines.

23 Si les sciences humaines jouent leur rôle normal, d'autres disciplines soutiennent fortement la réalisation de l'éducation pour une citoyenneté démocratique. C'est le cas des langues modernes et de l'éducation artistique : en fait, tout ce qui a un lien avec la création et les échanges culturels. Apprendre une langue étrangère, par exemple, signifie apprendre une culture, une autre manière de concevoir et comprendre le monde. En nous rapportant à celui - ci, nous pouvons construire notre propre conception sur le monde. 
L'étude des langues étrangères contribue à la formation des capacités de communication et indirectement d'aide pour l'intégration sociale des élèves, à l'éducation de ceux - ci pour une tolérance culturelle etc. Le Programme de Langue et littérature roumaine pour le collège, contient des objectifs coextensifs au nouvel idéal éducationnel : le développement d'une pensée structurée et de la compétence à solutionner les problèmes, la constitution d'un ensemble de valeurs concordant avec une société démocratique et pluraliste, la formation d'une attitude responsable, la formation des compétences de communication. Pour l'histoire, les élèves acquièrent des connaissances ayant trait au passé, des institutions aux formes de gouvernement et des régimes politiques, au rôle de l'Etat dans la vie de la société etc.

L'éducation se réalise seulement grâce à l'étude des disciplines contenues dans le Curriculum formel. La formation d'un citoyen apte à l'exercice de la démocratie est une exigence éducative fondamentale qui peut être réalisée dans une école organisée en fonction des principes démocratiques. Une école de telle sorte doit offrir un milieu propice au développement de la capacité des élèves à participer aux processus démocratiques, et l'effort qu'elle fait dans ce sens - là ne se réduit pas à ce qui est prévu dans le Curriculum formel. Par la multitude d'interrelations qui crée et stimule, ce type d'éducation favorise un apprentissage qui s'étend au - delà du cadre formel des activités curriculaires. Toute la structure organisationnelle de l'école est déterminée par son rôle essentiel, la réalisation du processus éducatif. Mais celle - ci exerce les influences éducatives sur les élèves tant par des actions directes, intrinsèques au processus instructif éducatif, que, par des actions indirectes, comme, par exemple, par l'intermédiaire du milieu scolaire, des activités extra curriculaires, l'organisation et la valorisation du partenariat avec d'autres facteurs éducatifs etc. L'éducation se réalise aussi par l'intermédiaire de la participation démocratique à la prise des décisions dans la classe (école), par l'ethos scolaire ou par le discours involontaire de la communauté scolaire: l'atmosphère, le sous conscient de groupe, les symboles dominants, les modalités de partage, les connaissances etc. L'association des élèves en vue d'organiser l'activité scolaire et la récompense de leur initiative en ce qui concerne le déroulement des activités extra scolaires sont capables de créer un cadre scolaire favorable à un tel comportement. En fait, la réalisation de l'éducation implique la coopération de tous les milieux d'enseignement qui forment le contexte scolaire. Autrement dit, l'école qui aura plus de réussites sera celle qui implique de façon permanente les élèves dans des activités efficientes non seulement au niveau individuel, mais aussi au niveau collectif. Elle doit être perçue comme une communauté où l'on peut apprendre le comportement démocratique, où les élèves se familiarisent avec différentes façons d'intégrer la communauté, en se rapportant continuellement aux valeurs comme la tolérance, la solidarité et la responsabilité. Une condition fondamentale pour participer aux processus démocratiques est représentée par le développement de l'esprit critique, du pouvoir de décision et de la pensée autonome. Indifféremment des niveaux d'étude, l'école doit être perçue comme l'endroit où se forme un public démocratique.

\section{Conclusions}

En se rendant compte de l'importance sociale qu'elle a, l'école fait des efforts pour se constituer dans une institution qui exerce la démocratie, en éliminant les pratiques autoritaires, de type totalitaire, en apprenant aux élèves à prendre des décisions à leur 
compte, à respecter les autres et à valoriser le résultat de l'interaction avec les autres, à comprendre et à suivre leurs propres intérêts, en tenant compte de ceux des autres, à agir en s'appuyant sur les principes démocratiques de liberté, responsabilité, égalité, justice, esprit communautaire etc. Les manifestations d'hyper direction et d'hyper contrôle venues de la part des enseignants ou de la part de la direction poussent les enfants vers le conformisme, vers l'adaptation passive. C'est pourquoi il est nécessaire de réaliser un climat scolaire permissif, centré sur une liberté assumée d'une manière responsable, sur la confiance et l'empathie.

L'école institue la citoyenneté, affirme P. CANIVEZ (1990) ; elle représente l'endroit où les enfants cessent d'appartenir exclusivement aux familles dont ils proviennent, afin de pouvoir s'intégrer dans une communauté plus large, où les individus sont réunis non par la parenté biologique mais par l'obligation de vivre ensemble. Autrement dit, l'école institue la cohabitation des différentes personnes sous l'autorité du même règlement, d'où la nécessité de transmettre aux enfants le respect pour un certain ordre qui les habitue à se soumettre à une organisation. Dès qu'ils s'habituent, ils commencent à obéir. En fait, il s'agit d'une question délicate car, dans l'école moderne, où les relations dominantes sont celles de la démocratie, l'esprit de liberté, l'autonomie et la responsabilité de l'individu constituent un idéal éducatif. Par la diversité et par le caractère actif des activités scolaires et extra scolaires, par les simulations et par le travail en équipe, on peut déterminer les élèves à avoir un esprit participatif et coopérant, ce qui constitue une qualité importante dans leur formation en tant que personnes et dans la perspective d'accomplir les tâches qu'il auront à finaliser plus tard.

\section{BIBLIOGRAPHIE}

ALMOND, Gabriel, Verba, Sidney (1996) Cultura civica, Editura DU Style, Bucuresti.

AUDIGIER, Françoise (1991) Enseigner la société, transmettre des valeurs, Conseil de la coopération culturelle, Strasbourg.

BRAMELD, Theodore, (1965) Education as power, Holt, Rinehart and Winson, New - York, London.

CANIVEZ, Patrice (1990) Éduquer le citoyen ? Édition Hatier, Paris.

DEWEY, John (1990) Démocratie et education, Armand Colin, Paris.

ENGLE, S. H., Ochoa, A. S. (1988) Education for democratic citizenship : Decision making in the social studies, New York, Teachers College Press.

HOUSSAYE, Jean (1992) Les valeurs à l'école. L'éducation aux temps de la sécularisation, PUF, Paris.

ROWE, David (1992) The Citizen and the Law. Teaching about the Rights and Duties of Citizenship, in : E. Baglin Jones, N. Jones (eds.), Education for Citizenship. Ideas and Perspectives for Cross - Curricular Study, London, Kogan Page.

VLASCEANU, Lazar (ed.) (2002) Scoala la rascruce. Schimbare si continuitate in curriculumul invatamantului obligatoriu, t. I, Editura Polirom, Iasi. 


\section{RÉSUMÉS}

Nous essayons d'offrir au lecteur une brève présentation du cadre formel dans lequel se réalise l'éducation pour une citoyenneté démocratique en Roumanie. La démocratisation continue de la société et le processus d'intégration européenne réclament impérativement une formation des individus centrée sur les valeurs universelles - le bien, la justice, la liberté et l'égalité mais aussi sur celles qui sont le résultat d'un système d'organisation démocratique de la société, où les droits de l'homme représentent des valeurs qui peuvent se transformer en comportements effectifs à travers l'éducation. Il s'agit de montrer comment ces valeurs sont présentées dans le système d'enseignement.

We will offer to the reader a short presentation of the formal frame in which the education for democratic citizenship is realized in Romania. The process of democratizing of the Romanian society and the process of European integration ask imperatively for an education focused on the universal values - good, justice, freedom, equity - and also on the values which result from the democratic organization of the society, in which the human rights are considered values, which can be transformed in effective behaviors only through education. We will present the way in which these values are promoted in the school system.

\section{INDEX}

Mots-clés : citoyenneté, curriculum

Keywords : citizenship, education

\section{AUTEUR}

\section{ION ALBULESCU}

Lecteur Dr. - Université Babes - Bolyai de Cluj - Napoca, Roumanie 